\title{
A micromechanical model and numerical simulation of framework interstice concrete
}

\author{
Q. G. Yang, Z. J. Yi, X. B. He, Y. H. Ma, F. Huang \& C. H. Zhao \\ Civil Engineering and Architecture School, \\ Chongqing Jiaotong University, P.R. China
}

\begin{abstract}
Being a kind of framework material, framework interstice concrete is composed of single-grade or gap-grade aggregate which are bonded by cement or bitumen, whose structural characteristic is "bonding crunode + aggregate + space". This paper develops a kind of micromechanical model, carrying out numerical simulation of flexual performance of the framework interstice concrete. The calculating results basically conform to the experimental result gained in the laboratory. The method discussed in the paper can be developed to apply in calculating analysis on all kinds of framework interstice concrete materials.
\end{abstract}

Keywords: framework interstice, framework interstice concrete materials, micromechanical model, numerical simulation.

\section{Introduction}

To accomplish a pavement's service functions, such as water permeability and noise reduction, framework interstice concrete material has been recently used more and more in pavements. In the perspective of its internal structure, cement or asphalt cementitious matter is used to bond single-grade or gap-graded aggregate to form a "bonding crunode + aggregate + space" internal framework structure. The framework formed by aggregates and elastic nodes endows materials with strength and deformation properties, and the connective space allows for its water permeability and noise reduction functions; therefore, framework interstice concrete material is a new kind of pavement material with good performance. 
Because of the existence and processing of inner structural space, it is difficult with this kind of material to build finite-element models and to simulate its mechanical performance.

Currently a variety of concrete micromechanical models have been established to research the relationship of concrete's micro-formation and its macro properties. In article by Tang [1], a plane model is used to analyze the concrete's properties. In the micro-models, the author considers the concrete as consisting of three sorts of materials - cement materials, aggregate and interface materials. When setting up models, the aggregates in fixed size are stochastically scattered in a certain plane area; the gaps among the aggregate are filled with cement material and the surface material with a certain depth is added as the third type of material outside the aggregate. The models were efficient to simulate the fracture process, especially when cracks emerge from the interface. For the properly-designed framework interstice concrete materials, cracks usually emerge in the bonding material, so there is no need for the third-phase material. In addition, it is hard to deal with the spaces in these models.

Adler et al. [2] proposes a method to deal with concrete internal voids from the viewpoint of geometry, but still much work needs to be done to build mechanical models. Articles [3-5] present a method of simulating the mechanical properties of asphalt bituminous concrete with the lattice model. In the micro lattice model, the aggregates are simplified as rigid joints, and the interaction of asphalt membrane among aggregates is replaced by a bar, whose mechanical parameter is gained by analyzing the asphalt membrane, and then a lattice structure can be established to simulate the performance of asphalt concrete. The advantage of the lattice model is that there is no need to consider the concrete's internal space and it's feasible to simulate the mechanical properties of concrete. But apparently, this kind lattice model cannot get the aggregate's influence on the property of the concrete, meanwhile, it cannot efficiently check the stress state of the bonding material. To some extent the performance of framework interstice concrete is decided by the bonding material, so both to investigate the stress state of the bonding material and to investigate the relationship between the bonding material and the whole concrete are important.

In order to research the performance of framework interstice concrete, this paper develops a micromechanical model, which can easily consider the spaces in framework interstice concrete and the effect of the aggregates. Based on the micromechanical model, the simulation results conform to that gained in the laboratory. The advantage of the method mentioned in the paper is that the study result of the stress state of bonding materials can guide the design of framework interstice concrete.

\section{The calculating principle}

Static finite element numerical calculation is to disperse the structure space and make displacement interpolation at the discrete nodes. 


$$
u_{i}(x, y, z)=\sum_{I=1}^{n} N_{I}(x, y, z) u_{i I}
$$

$i=1,2,3$ are indicators of spatial coordinates. $I=1,2, \ldots n$ are finite element node; $U_{i I}$ is the first element node in $i$ direction displacement, $N_{1(x, y, z)}$ is the first element of Lagrange interpolation function. We use elastic-plastic constitutive equation to calculate. The unit of the constitutive equation is written in form of matrix, geometric equation,

physics equation,

$$
\varepsilon=L u
$$

$$
\sigma=D \varepsilon
$$

balance equation,

$$
L^{T} \sigma+q=0
$$

stress boundary condition,

$$
L_{n}^{T} \sigma=p
$$

displacement boundary condition,

$$
u=\bar{u}
$$

elastic strain,

$$
\left\{\varepsilon^{e l}\right\}=\frac{\sigma^{e}}{E \varepsilon^{e}}\left\{\varepsilon^{n l}\right\}
$$

plastic strain,

$$
\left\{\varepsilon^{p l}\right\}=\left\{\varepsilon^{n l}\right\}-\left\{\varepsilon^{e l}\right\}
$$

elastic-plastic matrix,

$$
\left[D_{c p}\right]=\frac{\sigma^{e}}{E \varepsilon^{e}}\left[D_{c}\right]
$$

The elastic matrix of aggregate is gained by inserting anisotropic stress-strain relationship into isotropic materials. It can be expressed as:

$$
[D c]=\frac{E}{(1+v)(1-2 v)}\left[\begin{array}{cccccc}
(1-v) & v & v & 0 & 0 & 0 \\
v & (1-v) & v & 0 & 0 & 0 \\
v & v & (1-v) & 0 & 0 & 0 \\
0 & 0 & 0 & \frac{(1-2 v)}{2} & 0 & 0 \\
0 & 0 & 0 & 0 & \frac{(1-2 v)}{2} & 0 \\
0 & 0 & 0 & 0 & 0 & \frac{(1-2 v)}{2}
\end{array}\right]
$$

$\left\{\varepsilon^{n l}\right\}$ is the general strain vector, $\left\{\varepsilon^{e l}\right\}$ is strain vector. $\left\{\varepsilon^{p l}\right\}$ is plastic strain vector, $\left[D_{c}\right]$ is elastic matrix, $\left[D_{c p}\right]$ is plastic matrix, $L$ is differential operator, $D$ is rigid matrix, $q$ is volume force vector, $p$ is surface force vector. 


\section{The micromechanical model of framework interstice concrete}

\subsection{Geometric model}

This article adopts a plane model to simulate the mechanical properties of the framework interstice concrete.

Because the framework interstice concrete's features and force carrying characteristic are dependent on its inner structural constitution "aggregate + elastic nodes + void", in general, the aggregate size of the framework interstice concrete only influences the space size, but little to the main performance of the framework interstice concrete.

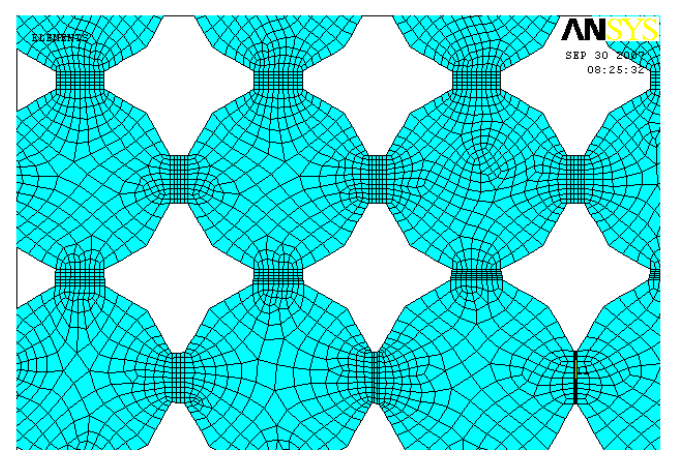

Figure 1: The shape and arrangement of aggregate and bonding material.

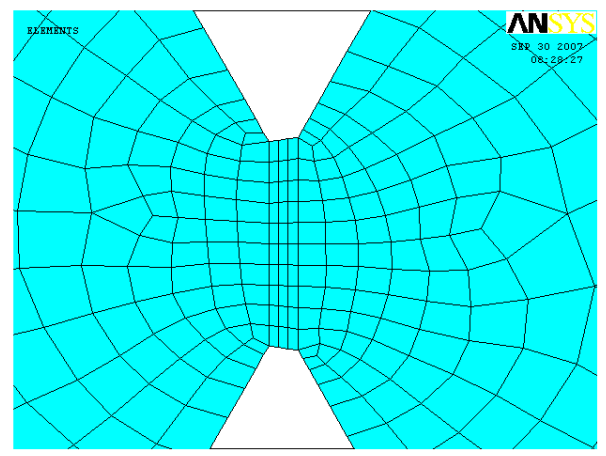

Figure 2: The mesh of aggregate and bonding material.

During calculations, when the bonding material is more cured, the aggregate can be simplified to a single size. In the model, the aggregate shape is treated as a twelve-edged polygon with same long sides for the convenience of modeling (Figure 1). Accordingly, the gaps between two aggregates are filled with bonding material evenly. 
The advantages of this kind of modeling are: when the aggregate is simplified, the aggregate's adjacent edges are straight, and it is easy to get bonding material mesh without an abnormal unit (Figure 2); moreover, the other gaps which are not filled with bonding material left on the geometry can be regarded as the natural spaces of the framework interstice concrete to achieve the physical similarity (Figure 1). In this way, one can focus more on the bonding material in the calculation, at the same time, pay attention to the relationship between the bonding mechanical parameter and the overall performance of framework interstice concrete.

\subsubsection{Aggregate}

Aggregate size: diameter unified as $5 \mathrm{~mm}$. In practice, framework interstice concrete usually adopts gap-graded or single-graded aggregate; considering the focus of the calculation is to find the bonding's effect on the overall performance of the framework interstice concrete and to simplify the model, the aggregate size is unified as $5 \mathrm{~mm}$ which is near to the actual size. Meanwhile the smaller the aggregate size is, the more convenient to get befitting mesh of the two different materials - aggregate and bonding material.

Aggregate shape: when the aggregate is simplified as circular, the circle of two aggregates will lead abnormal mesh and stress singularity (Figure 1, 2). Therefore, it is simplified into a twelve-edged polygon with the same surface.

\subsubsection{Bonding material}

The bonding thickness between aggregate is $0.1-0.5 \mathrm{~mm}$ according micromeasure. In usual, the bonding thickness is $0.5 \mathrm{~mm}$, so in the model the bonding material thickness is chosen as $0.5 \mathrm{~mm}$ (Figure 2).

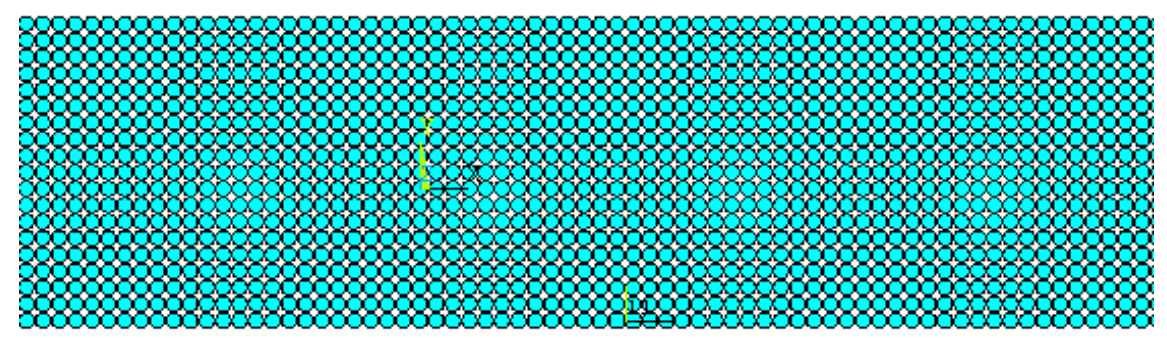

Figure 3: The overall model of framework interstice concrete.

\subsubsection{The overall model}

Aggregate is in a parallel arrangement and only the gaps between two adjacent parallel edges are filled with bonding material (Figure 1,2), while the other parts aren't filled, the rest of the parts simulating the natural spaces.

These two kinds of materials in accordance with the planar combination eventually form $10 \times 40(\mathrm{~cm})$ planar pieces (Figure 3 ) so that we can compare the results with the $10 \times 10 \times 40(\mathrm{~cm})$ bending experimental test. 


\subsection{Unit analysis}

Unit type: because of the difficulties and the feasibilities of the threedimensional model, we choose the plane model which can consider thickness for analysis. ANSYS' PLANE42 is able to establish two dimensional entity structural models. The unit can be used as both a plane unit and axisymmetric unit. At the same time the unit can deal with plasticity, creep, large deformation and large strain problems. The overall model of framework interstice concrete has $1,109,723$ units and 1,182901 nodes.

\subsection{The model mesh}

Bonding material: it can be divided into three units in thickness, ten units in length, and the unit's ratio between width and length is $1 / 5$, that accord with the basic requirement of units (Figure 2).

The aggregate: the adjacent part of aggregate and bonding coordinates with the bonding material's unit, while other parts divide by themselves according to the principle of adapting. Aggregate and its units dividing is illustrated in Figure 1.

\subsection{Material mechanical parameter}

\subsubsection{Aggregate's mechanical parameter}

The aggregate material used in the test is granite, whose elastic modulus is 50$85 \mathrm{GPa}$. According to the test and related test data, the elastic modulus in the calculation is 60Gpa. Granite Poisson's ratio is between 0.20 and 0.30 in test, and it is 0.27 in the calculation. Granite Stress-strain relationship is linear.

\subsubsection{Bonding material mechanical parameter}

In this paper, the bonding material used in the test is polymer cement material, whose parameters are from the actual test data in the laboratory. The following figures are two representative curves of the polymer cement measured in the test.

Through experiments, it is found that the bonding material elastic modulus is between 7000 8000Mpa (Figure 4). So it is 7500Mpa in the calculation

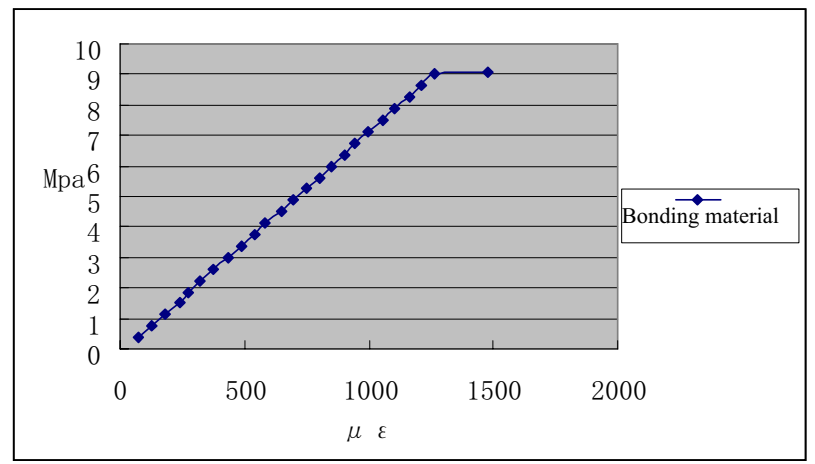

Figure 4: Bonding material stress-strain curves from experiments. 


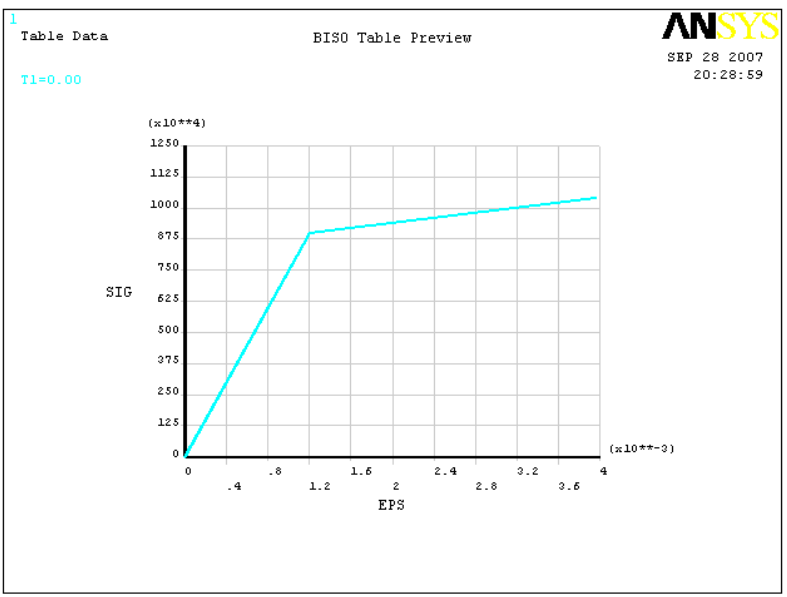

Figure 5: Bonding material stress-strain curves in the calculation.

(basically one degree lower than that of aggregate) and the Poisson's ratio is 0.23. According to experiments its stress-strain relationship is chosen as typical dual line (Figure 5).

The first segment of the straight line, as in the test: the yield stress can be looked on as 9Mpa, and we choose the straight line before the stress reaches $9 \mathrm{Mpa}$; the second segment: it is a little slope so as to avoid the dis-convergence.

\subsection{Boundary condition and loading}

Linear loading is adopted in the calculation. Loading location and the supporting condition is in accordance with that in the experiment (Figure 6).
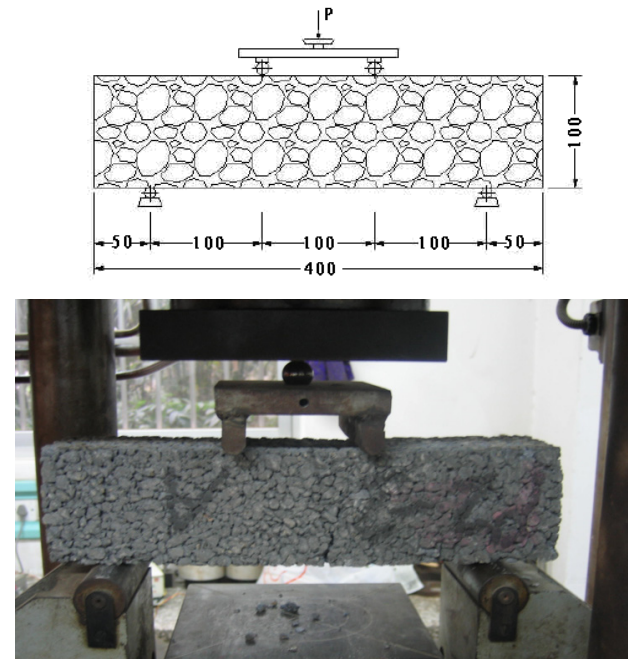

Figure 6: Schematic diagrams of loading (unite: $\mathrm{mm}$ ) and loading photo. 


\subsection{Solution method}

The paper adopts Newton-Raphson method to solve the equations. The finite element formulation of Newton-Raphson divides the entire load-displacement process into a series of incremental segments; and in each increment, the structure's loading response is nearly as linear. Besides, after each increment's load is increased, structural tangent stiffness matrix would be modified many times according to the required status variable to eliminate the unbalanced force and make sure that the calculation results satisfy the given precise requirement. And then considering the status as the equilibrium state, we continue to act on the loading increment to get incremental displacement by solving linear algebraic equations.

\section{Calculation results analysis of framework interstice concrete}

\subsection{The simulated results of flexural test}

After the simulated flexural specimen, which is loaded according to Figure 6, the framework interstice concrete's mid-span load-displacement curve is gained. (Figure 7.)

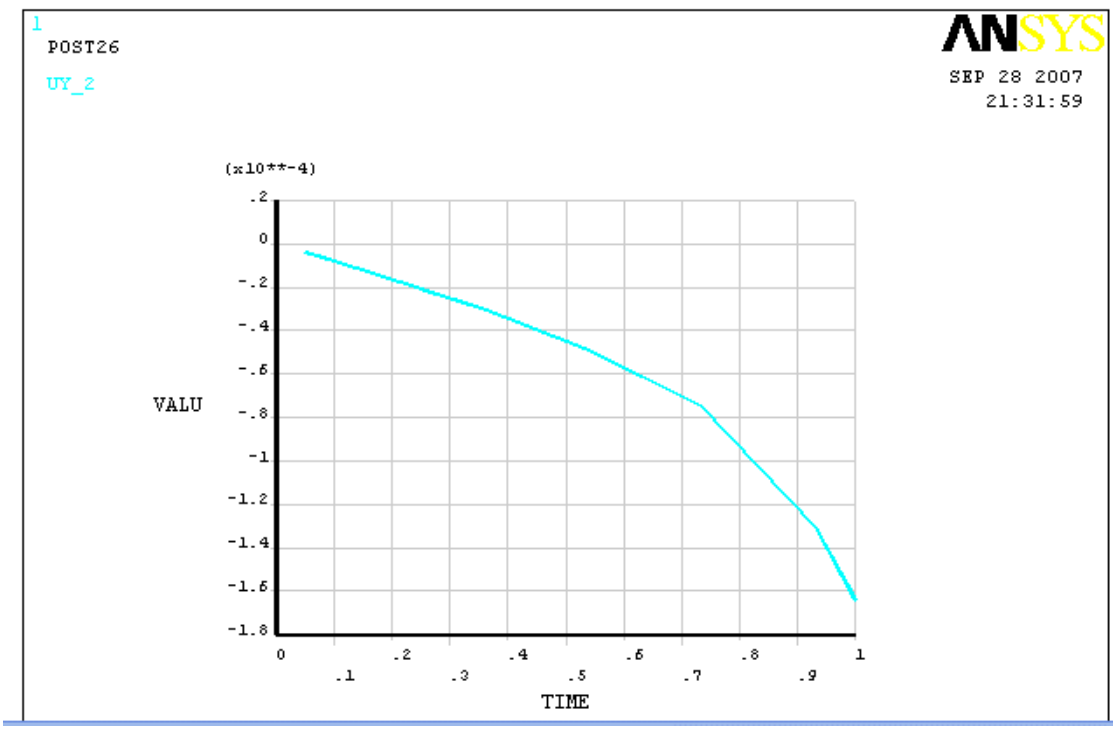

Figure 7: Framework interstice concrete's mid-span load-displacement curve.

The calculated curve shows when the load is added to $14.6 \mathrm{KN}$ (the load is two times of TIME STEP in ANSYS), the framework interstice concrete appears yielding, at this time, the corresponding displacement is $0.075 \mathrm{~mm}$. 


\subsection{Experimental results}

Figure 8 is an actual measuring stress-strain curve, and in test the measuring point is at mid-span bottom of the concrete beam. From the chart: when the load is increased to $12.3 \sim 15.2 \mathrm{KN}$ (in the loading as Figure), the concrete stress at mid-span bottom has a specifically relation with loading, $\left.\sigma=P L /\left(b h^{2}\right)\right)$, the framework interstice concrete appears yielding.

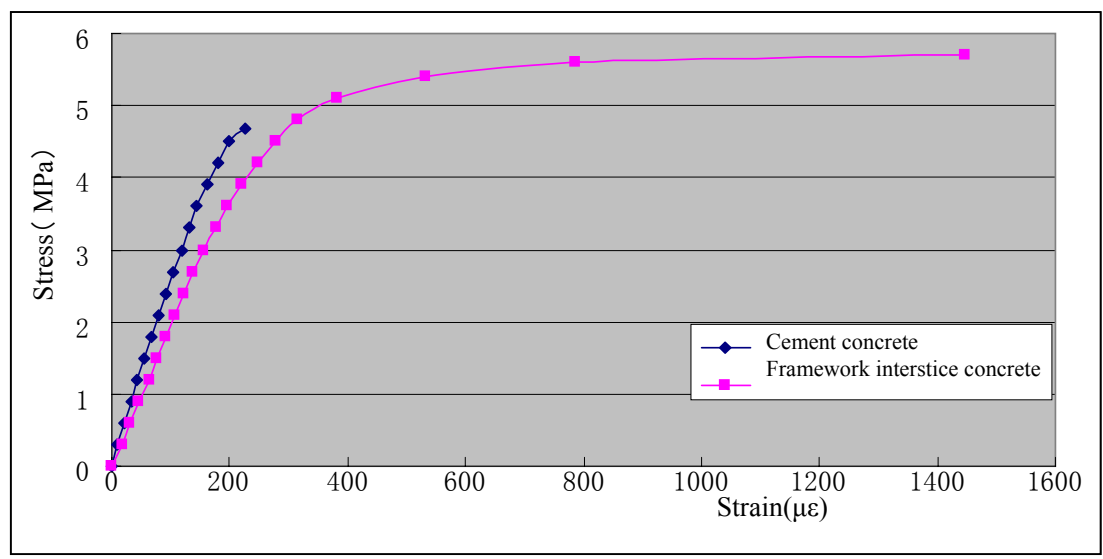

Figure 8: The representative stress-strain curve of framework interstice concrete (the measuring point is at mid-span, bottom of the concrete beam)

\subsection{The comparison of the calculation result and experimental test}

Comparing the result of test with that of calculation, the calculation yielded load has $5 \% \sim 18.7 \%$ difference with the actual yielded load in experimental test, but the mid-span displacement has about $30 \%$ difference with the actual (it is maybe because the support has local deformation). From the comparison, conclusions can be drawn: the calculation model in the paper can better simulate the framework interstice concrete's mechanical properties.

\section{Conclusions}

The micromechanical calculation model developed in the paper, with a clear concept and convenient modeling, can better forecast and analyze the mechanical properties of framework interstice concrete, whose calculation results are consistent with that in the laboratory. The method in the paper can further simulate and study the mechanical actions of framework interstice concrete under other loading conditions, and popularize to study the creep of bituminous concrete materials. Henceforth, the model can be further developed into a three 
dimensional model in order to better simulate framework interstice concrete materials' mechanical features.

\section{Acknowledgements}

The study has been supported both by Ministry of Transport of the People's Republic of China through project (2008 318 814 62) and by Chongqing Science \& Technology Commission projects, whose support is gratefully acknowledged.

\section{References}

[1] Tang C. A., Numerical tests of progressive failure in brittle materials. Journal of Mechanics and Practices, 21(2), pp. 21-24,1999.

[2] Adler. P.M, Thovert. J.F, Bekri .S \& Yousefian. F, Real Porous Media: Local Geometry and Transports. Fracture. Journal of Engineering Mechanics /August, pp. 829-839,2002.

[3] Arslan A., Ince R. \& Karihaloo B.L., Improved Lattice Mode for Concrete Fracture. Journal of Engineering Mechanics/January, pp. 57-65, 2002.

[4] Gianluca Cusatis, Zdenek P. Bazant \& Luigi Cedolin, Confinement-Shear Lattice Model for Concrete Damage in Tension and Compression: I. Theory. Journal of Engineering Mechanics ASCE/December, pp. 1439-1448,2003.

[5] Dai Q.L., Martin H. Sadd, Venkit Parameswaran \& Arun Shukla, Prediction of Damage Behaviors in Asphalt Materials Using a Micromechanical FiniteElement Model and Image Analysis. Journal of Engineering Mechanics ASCE/July, pp. 668-676,2005. 